\title{
Thyroid Fine-Needle Aspiration Cytology:
}

Causes of false-positive results

\section{Daniela C Malheiros ${ }^{1,2^{*}}$, Sule Canberk ${ }^{2,3,4^{*}}$, David N. Poller ${ }^{5}$ Fernando Schmitt ${ }^{2,3,6}$}

1. Faculdade de Ciências Médicas da Santa Casa de São Paulo, Brasil

2. IPATIMUP, Institute of Molecular Pathology and Immunology of University of Porto,

$$
\text { Porto, Portugal }
$$

3. I3S, Instituto de Investigação e Inovação em Saúde, University of Porto, Porto, Portugal

4. Department of Pathology subdivision of Cytopathology, Acibadem University, Kayısdag1 cd. Atasehir-Istanbul-TR, Turkey

5. Department of Pathology, Queen Alexandra Hospital, Cosham, Portsmouth, PO6 3LY, UK 6. Medical Faculty of Porto University, Porto, Portugal

* Both authors contributed equally as first authors.

\section{Correspondent author:}

\section{Fernando Schmitt, MD PhD, FIAC}

Instituto de Patologia e Imunologia Molecular da Universidade do Porto (IPATIMUP),

Rua Julio Amaral de Carvalho 45

4200-135, Porto, Portugal

e-mail address: fernando.schmitt@ipatimup.pt 


\section{$\underline{\text { Abstract }}$}

In this paper, we aim to focus on false positive results in the evaluation of thyroid aspirations, covering cystic, inflammatory, follicular and oncocytic lesions, papillary carcinoma, and medullary carcinoma of thyroid. The recently described entity, "noninvasive follicular thyroid neoplasm with papillary-like nuclear features" (NIFTP), is also discussed detailing the impact of its introduction on the sensitivity and specificity of thyroid FNA, as well as the use of molecular tests for diagnostics. Medicolegal issues in relation to current practice in English law are also described.

Keywords: thyroid FNA, False-positive, NIFTP, Follicular-pattern lesions, thyroid carcinoma 


\section{$\underline{\text { Introduction }}$}

Fine-needle aspiration cytology (FNAC) is widely accepted as a rapid, cost-effective, and safe method for the diagnosis of thyroid nodules although it is accepted that false positive diagnoses of malignancy do occur. ${ }^{1,2}$ The positive predictive value of FNAC for malignancy according to The Bethesda System for Reporting Thyroid Cytopathology (TBSRTC) is 97\%99\%, and this figure includes NIFTP tumours, the sensitivity and specificity of thyroid FNAC have been reported as $65 \%-99 \%$ and $72 \%-100 \%$, respectively. ${ }^{1-4}$ The Royal College of Pathologists in its 2016 Guidance on the Reporting of Thyroid Cytology Specimens also indicates from review of the published literature a sensitivity for malignancy typically of $65 \%$ to $98 \%$, a specificity of $76-100 \%$, and a false positive rate of $0-5 \%$. The results of fifth UKwide national audit of thyroid surgical practice conducted by The British Association of Endocrine and Thyroid Surgeons (BAETS) shows a false positive rate for Thy5 (equivalent to Bethesda Category VI) of around 2\%. The BAETS National Audit does not include nonoperated cases. The rates of cancer diagnosis of all types recorded in The BAETS Fifth National Audit were Thy 5 (equivalent to Bethesda Category VI) 97.9\% (1,414 / 1,444 cases) and Thy 4 (equivalent to Bethesda Category V) 74.4\% (623 / 837 cases) ${ }^{6}$.

The main goal of thyroid FNAC is to determine the presence or absence of a neoplasm, then to diagnose any neoplasm present as benign or malignant, and finally to determine the exact type of tumour present. ${ }^{2,4,7}$ Thyroid FNAC can be used as a 'rule in' test most of the time for papillary carcinoma but for follicular and medullary carcinomas for example, thyroid cytology functions as a 'rule out' test much of the time. In adults, FNA has been established in large reviews as a useful test in the differentiation of a thyroid nodule as malignant or benign. ${ }^{8,9}$ Many research groups have found that FNA in children and adolescents is a highly sensitive and accurate test in differentiating benign from malignant nodules. ${ }^{10-13}$ In a study 
by Stevens et al, sensitivity and specificity of this test in the pediatric population were $94 \%$ (95\% confidence interval $[\mathrm{CI}], 86 \%-100 \%$ ) and $81 \%$ (95\% CI, 72\%-91\%). ${ }^{14}$ However, misdiagnosis of cancer by cytopathology examination has remained one of the most problematic issues in thyroid pathology. The rate of false-positive FNAC for thyroid nodules has been reported as $2 \%-10 \%,{ }^{3,15}$ compared with rates of $0 \%-14 \%$ for false-negative results 16,17. The mean risk of malignancy for lesions classified as suspicious for malignancy and malignant in TBSRTC is $75.2 \%$ and $98.6 \%$, respectively. Overdiagnosis resulting from cytopathology results may lead to excessive treatment, specifically, unnecessary thyroidectomy and also other treatments. Considering the current trend towards conservative treatment for patients with thyroid carcinoma, false positivity can be as problematic as false negativity. In particular, thyroid surgeons could face the experience of performing unnecessary surgery in patients with false positive results. ${ }^{3}$ In this paper, we discuss the main causes of false positive in thyroid FNAC (Table 1). We divided the lesions in two groups: cystic and solid.

\section{Cystic Lesions}

The term cystic thyroid nodule is used to describe any fluid-filled thyroid nodule. Approximately $15 \%$ to $25 \%$ of solitary thyroid nodules are cystic or predominantly cystic, and most nodules are benign. ${ }^{18}$ Despite the good performance of FNAC in the evaluation of thyroid nodules, its accuracy is lower in cystic lesions than in solid masses ${ }^{19-21}$ Cystic lesions of the thyroid constitute a particular problem in FNA especially because malignant neoplasms may also present as a simple or complex cyst. The potential for false-negative diagnosis of cystic thyroid malignant neoplasms is well demonstrated in the literature. ${ }^{1,2,4}$ However, cystic lesions also can be a cause of false positive diagnosis. Cyst-lining cells are follicular or mesenchymal cells with reactive changes, associated with cystic degeneration of thyroid 
nodules. The reactive cyst lining cells have distinct cytoplasmic borders, loose to dense granular cytoplasm, occasional exaggerated unipolar/ bipolar cytoplasmic processes (Figure 1). Nuclei can be large, round to oval but always with regular borders, and may show grooves and hypochromasia with distinct nucleoli. ${ }^{2,4,22}$ The spindle-shaped morphology of the cells, resembling reparative epithelium in cervical, bronchial and gastrointestinal cytological specimens is useful in distinguishing these cells from papillary thyroid carcinoma (PTC). In rare instances these cells are more packed, less elongated and with overlapping, making it more difficult to rule out PTC. According to TBSRTC, a diagnosis of atypia of undetermined significance (AUS)/ follicular lesions of undetermined significance (FLUS) is more appropriate.

Cystic changes and degenerative processes may cause florid atypia which is a significant cause of false positive and negative results. In a study on thyroid cysts containing atypical cells, among 12 selected cytological features, intranuclear pseudoinclusions, papillary microarchitecture, nuclear crowding and mitoses were the ones suggesting carcinoma. ${ }^{22}$ Because of some studies suggesting that atypical cells can be the cause of false positive repeat FNA over a short period of time, the previous edition of TBSRTC suggested to wait at least 3 months before repeating the FNA, however, the 2017 edition of TBSRTC did not accept the evidence for the effect of early repeat FNA on increased atypical results ${ }^{2}$. Histological alterations can occur following thyroid FNAC, such as infarction, necrosis and vascular changes. These alterations are not frequent but can cause false-positive histological diagnosis. Thin needles and a non-aspiration FNAC technique can reduce these findings. ${ }^{23}$

Squamous metaplasia can be also a source of a false-positive diagnosis. Cystic lesions of congenital rest origin, such as intrathyroidal lymphoepithelial cysts of probable branchial cleft origin are often sources of intrathyroidal squamous cells. The FNA specimens from these cysts consist of macrophages, neutrophils, and a mucoid or proteinaceous background 
admixed with squamous cells. ${ }^{24}$ In some cases, the squamous metaplasia can be so extensive that may lead to the suspicion of PTC with squamous differentiation. ${ }^{24}$ If atypical cytologic features are appreciated in a smear with a mixed pattern (few squamous cells, follicular cells and colloid, plus/minus inflammatory cells and macrophages), reactive squamous metaplasia arising in the background of cystic change or Hashimoto thyroiditis is the most likely explanation. ${ }^{24}$

In some situations, performing a cell block in a cystic aspirate can convert nondiagnostic results into a satisfactory sample, as well avoiding false-positive results. ${ }^{25}$

\section{$\underline{\text { Inflammatory Lesions }}$}

Inflammatory lesions of thyroid include acute, granulomatous thyroiditis and lymphocytic thyroiditis (Hashimoto's thyroiditis). FNA is indicated in these situations when by palpation or imaging a nodule is identified.

\section{Acute suppurative thyroiditis}

Tenderness, fever, debility and high ESR should enable a confident clinical diagnosis of acute thyroiditis, but the condition may resemble a rapidly growing anaplastic carcinoma, since the latter is frequently necrotic and accompanied by neutrophils. Sometimes in anaplastic carcinoma, the inflammatory infiltrate is so florid that it obscures the malignant cells. The malignant cells may also have a histiocytic or fibroblastic appearance, enhancing the possibility of confusion with acute suppurative thyroiditis. Therefore, in the presence of histiocytes and neutrophils, we need to give a differential diagnosis, but the definitive diagnosis of anaplastic carcinoma requires the identification of clearly malignant cells. The presence of bacteria (MGG, Gram stain) in thyroiditis or bizarre giant cells and obvious 
features of malignancy in carcinoma allows a correct diagnosis. The response to antibiotics should be dramatic in infective lesions.

\section{Granulomatous Thyroiditis}

Granulomatous reaction due to subacute thyroiditis (De Quervain's thyroiditis) is much more common than other granulomatous conditions of thyroid such as fungal infections, tuberculosis or sarcoidosis, ${ }^{4,26}$ comprising nearly $3-6 \%$ of all thyroid lesions. ${ }^{26}-28$ Localized anterior neck pain, glandular tenderness and diffuse pain in the ears are the usual clinical features. Although the diagnosis is usually made clinically, FNAC plays a pivotal role in differentiating De Quervain's thyroiditis from other lesions mimicking it, specifically malignancies and other forms of thyroiditis. ${ }^{29}$ Numerous neutrophils, and mononucleated inflammatory cells are present in early stage while an increased amount of fibrous stromal fragments and abundant multinucleated giant cells mixed with lymphocytes are much more prominent in the later stages. Follicular epithelial cells are usually scanty, frequently have small vacuoles in the cytoplasm and may show some degree of atypia; in addition, epithelioid histiocytes may remain unrecognized and can be interpreted as atypical cells, especially in cases with smearing artefacts (Figure 2). Cell debris in the background of smears can also simulate necrosis (Figure 3). Recognizing the cytomorphological details of multinucleated giant cells, epithelioid histiocytes, and fibrotic degenerate stromal fragments in the busy background created by acute-chronic inflammation and debris is important to prevent false positive diagnosis in this entity. ${ }^{4,26}$

\section{Lymphocytic Thyroiditis/Grave's Disease}

Hashimoto's thyroiditis is a well-established entity with typical clinical and cytomorphological findings. In the initial phase, there is antibody-mediated destruction of 
thyroid follicles and lymphocytic infiltration. In the chronic phase, there will often be only minimal residual and atrophic follicles with fibrosis of the thyroid parenchyma. ${ }^{30}$ Polymorphic lymphoid infiltration, plasma cells, micro-tissue fragments with germinal center formation, and oncocytic changes in the epithelium are typical features. Pitfalls in the cytological evaluation vary depending on the stage of the disease as well as accompanying Graves' disease. The "cellular stage" is composed of a proliferation of oncocytes. Oncocytic change leads to some nuclear atypia: nuclear enlargement, fine chromatin texture, prominent nuclear membranes and macronucleoli (Figure 4). Nuclear grooves and even pseudoinclusions are described in Hashimoto's thyroiditis. ${ }^{3,4,31}$ Not only these changes but also squamous metaplasia of thyroid follicular epithelial cells in "fibrotic stage" may give a misleading impression as suspicious for malignancy. Indeed, Hashimoto's thyroiditis is one of the most common causes of false positive PTC diagnosis. ${ }^{3,4,31}$ In a recent study, involving 3048 cases of PTC it was detected in 48 false positive cases. ${ }^{3}$ Among these 48 patients with false-positive cytology results, final surgical specimens were positive for thyroiditis in 26 (54.2\%), which is a very high rate compared with the rate of pathological thyroiditis $(9.4 \%)$ among the total number of patients finally diagnosed as PTC. When cases of clinical thyroiditis were included, the ratio was further increased. These findings support the possibility that thyroiditis is a risk factor for false positivity of FNAC. ${ }^{3}$ On the other hand, PTC arising in Hashimoto's thyroiditis" is not rare; and a cytomorphologically clear-cut PTC should not be downgraded as 'atypia' or 'suspicious for malignancy' because of the presence thyroiditis in the background. Besides, "Warthin's variant of PTC" should also be remembered and excluded in an aspirate composed of oncocytic cells and lymphocytes. In a study by Harvey et al., it was shown that epithelial groups of Hashimoto's thyroiditis are usually infiltrated by lymphocytes in contrast to the epithelial clusters of PTC, which are usually devoid of lymphocytes. ${ }^{31}$ 
Sampling dominant nodules in Hashimoto's thyroiditis may produce aspirates mainly composed of oncocytes in macrofollicles, microfollicles, trabeculae, or solid sheets that could be interpreted as an oncocytic follicular neoplasm leading to a lobectomy. ${ }^{4,31}$ Careful search for the lymphocytic component in the background is necessary; even if it is lacking, in the appropriate clinical setting TBSRTC recommends to report such cases AUS/FLUS instead of "Follicular neoplasm/Suspicious for follicular neoplasm (FN/SFN)". ${ }^{2}$ Aspirates in the "florid lymphoid phase" may be entirely composed of lymphocytes and may be confused with lowgrade lymphoma (Figure 5). Detailed clinical findings and flow cytometry would be very useful in this differential diagnosis. ${ }^{32}$ Serology testing for thyroid autoantibodies should be part of the workup of cases of lymphocytic thyroiditis and in presence of a positive autoantibody test in an atypical cytology the risk of malignancy differs if the test is negative.

Graves' disease is a common cause of hyperthyroidism and reported rates of malignancy vary between 2.3-19\%. ${ }^{4}$ Oncocytic and/or non-oncocytic thyroid follicular epithelial cells,large amounts of flame cells, watery colloid, lymphocytes, and rarely granulomas are seen in aspirations. ${ }^{33}$ Cytological findings are challenging, especially after treatment. ${ }^{34}$ Distinctive pleomorphism, hyperchromasia, elongation of nuclei with occasional grooves and inclusions can be seen in this entity. ${ }^{4,33,34}$ To diagnose PTC in the background of Graves $^{\prime}$ disease, all the PTC criteria should be present in the aspirates. ${ }^{33}$

\section{$\underline{\text { Follicular-pattern Lesions }}$}

The main diagnostic challenge of FNA is what to do with the finding of follicular cells on FNA cytology. ${ }^{35}$ The range of follicular lesions of the thyroid includes adenomatoid nodule (AN), follicular adenoma (FA), and follicular carcinoma (FC) with the presence or absence of a capsule defining the histopathological differences (AN vs. FA) and capsular/vascular invasion (FA vs. FC). ${ }^{4,36}$ For these lesions, by definition, cytopathology is a 
screening test rather than a diagnostic test. FN/SFN is the category covering these lesions in TBSRTC. ${ }^{2}$ Apart from AN, FA and FC, follicular variant of papillary carcinoma (FV-PTC), poorly differentiated carcinoma, parathyroid adenoma, dyshormonogenetic goitre, and thyroiditis are the other entities that could be considered in the differential diagnosis in this category. Follicle-pattern lesions can appear in cytology as micro or macrofollicles. Microfollicles are composed of less than 15 overlapping thyroid follicular epithelial cells forming a circle that is at least two-thirds complete with or without intraluminal dense colloid. 37 Macrofollicles, form small or large flat sheets/groups or even rows but not circles; overlapping is not their character. Microfollicles can be observed as primary pattern- scattered isolated single microfollicles- or in groups as secondary pattern. In bloody smears, the presence of clots can create pseudo microfollicular appearance and we should be careful not to call the crowded small groups in clot microfollicles (Figure 6). In this situation, observing microfollicles out of the clot may be helpful. ${ }^{2,4}$ Sampling from microfollicular/cellular areas of benign follicular nodules may trigger a diagnosis of FN/ SFN and give rise to unnecessary surgery. However, the main goal of cytopathology in these lesions is not to establish an absolute diagnosis but the correct management for the patient. Follicular variant PTC (FVPTC) must be excluded from FN/SFN category. The nuclei of follicular lesions/neoplasms are expected to be round and rather hyperchromatic with coarse granular chromatin, which might be slightly larger than regular thyroid follicular epithelial cells. It is well known that some of the FVPTCs do not show the characteristic nuclear features of PTC and may present with subtle nuclear changes. The cytologic diagnosis of malignancy in these cases is based primarily on the nuclear features with or without the overall architectural information. The introduction of the entity NIFTP as mentioned before creates the possibility of false positive diagnosis of PTC on cytology specimens. ${ }^{38-41}$ This entity and its impact on routine FNA practice are discussed later. 
Intrathyroidal parathyroid adenoma and dyshormonogenetic goitre are the other entities that are characterized by a microfollicular pattern and lack of colloid. Noting presence of a clinical history of congenital hypothyroidism in dyshormonogenetic goitre and performing PTH assays on needle rinses or immunohistochemical studies on cell blocks are good approaches to the differential diagnosis. Microfollicles without intrafollicular colloid (empty follicles) and presence of large bizarre cells is highly suggestive for dyshormonogenetic goiter. Parathyroid adenomas show a prominent vascular network and neuroendocrine type chromatin. Epithelial clusters or microfollicles may predominate in the aspirates and many bare nuclei are commonly present in the background. Particularly for aspirates from thyroidectomy beds, which show a microfollicular pattern, parathyroid tissue should be remembered in the differential diagnosis. ${ }^{42}$

Hypercellularity, a microfollicular pattern, prominent atypia, and bizarre cells with big-dark nuclei can also be seen in thyroid after radiation (radioactive iodine/ external radiation beam therapy) and/ or some medical therapies (anti-thyroid, chemotherapy) and may mimic malignancy. ${ }^{4,} 34$ Clinical information about previous therapy is fundamental for the correct diagnosis when these morphological findings are present.

PTEN hamartoma tumour syndrome (PTHS) encompasses different syndromic disorders which are all associated with a germline mutation (point mutation or deletion/duplication) of the tumour suppressor gene PTEN, including Cowden Syndrome. Recent data suggest that thyroid disease in patients with PTEN mutation occurs at an early age in life. The high percentage of thyroid lesions at the first ultrasound after diagnosis of PTEN mutation underlines the importance of screening for thyroid disease immediately after diagnosis of PTHS. The high percentage (75\%) of thyroid pathologies in total emphasizes the importance of a structured follow-up, including FNAC. Most of these lesions are follicularpattern lesions. ${ }^{43}$ 


\section{$\underline{\text { Oncocytic Lesions }}$}

Oncocytic lesions of thyroid present a spectrum from hyperplasia / metaplasia to neoplasia. Oncocytic/Hurthle cells have abundant, finely granular, dense eosinophilic cytoplasm, and centrally placed large round nuclei with prominent nucleoli. Not only does it describe a phenotype but it also identifies a unique biologic process: the proliferation of morphologically abnormal mitochondria in the cytoplasm of neoplastic cells. ${ }^{44}$ Oncocytes and hyperfunctioning thyroid follicular epithelial cells may look similar at first glance; however, oncocytes have defined cell borders and granular cytoplasm. Pitfalls in the diagnosis of oncocytic lesions are quite similar to those seen in follicular lesions and can be summarized in three parts; -the differentiation between non-neoplastic and neoplastic lesions, -true oncocytic neoplasia versus oncocytic variant of other thyroid malignancies and oncocytic adenoma versus carcinoma which is not possible by cytology. Background colloid, inflammation and the ratio of oncocytic cells are important to identify non neoplastic nodules and to avoid falsepositives; the aspirates from oncocytic neoplasms maybe composed of $>70 \%$ oncocytes. ${ }^{45} \mathrm{In}$ a series of 70 cases of thyroid nodules with Hurthle cells (HC), Kasper et al. ${ }^{46}$ described 4 false-positive cases of Hurthle Cell Neoplasia (HCN). Three cases were multinodular goiter with nodules composed predominantly by HCs and one case was a Hashimoto thyroiditis. Overall high cellularity, scant or sticky colloid and $>90 \%$ HCs on FNAB are consistently seen in a neoplastic HC process, although are not sufficient to distinguish benign from malignant. In instances when high cellularity and a high percentage of $\mathrm{HCs}$ are associated with the presence of abundant colloid and/or a background of Hashimoto's thyroiditis, these lesions are better classified as follicular lesions or HCs of uncertain significance rather than as suspicious or positive for $\mathrm{HCN}$. A finding of prominent nucleoli is more commonly seen in a neoplastic process. However, the presence of prominent nucleoli on a background of 
Hashimoto's thyroiditis is to be interpreted with caution in order to avoid overdiagnosing these nodules as neoplastic. The follicular and oncocytic variants of PTC also remain diagnostic challenges. ${ }^{46}$

\section{Noninvasive Follicular Thyroid Neoplasm with Papillary-like Nuclear Features (NIFT-}

P)

Recently a subset of encapsulated FV-PTC with a very indolent clinical course was defined when treated with lobectomy alone (without radioactive iodine treatment). This new entity was called "noninvasive follicular thyroid neoplasm with papillary-like nuclear features" (NIFTP) and there are specific criteria for this diagnosis. ${ }^{38}$ Since this lesion is considered "a very low risk malignancy" but it shares some cytological findings with PTC, it should also be considered a potential cause of false positive results in thyroid FNA. ${ }^{39}$ The challenge posed by NIFTP is especially with the "suspicious for malignancy" and "malignant" categories, where the FNA diagnosis can frequently prompt initial total thyroidectomy, but where more conservative management (i.e. lobectomy) would be preferable if the histological diagnosis proves to be NIFTP. ${ }^{38}$ The effects of reclassification of some cases of FV-PTC as NIFTP on the risk of malignancy (ROM) associated with the categories of TBSRTC are minimal if strict criteria are used. ${ }^{39,40,41}$

To avoid false positive FNAC, the six criteria for PTC recommended by TBSRTC are required for a definitive diagnosis of malignancy: enlarged nuclei, oval or irregularly shaped and sometimes moulded nuclei, longitudinal nuclear grooves, intranuclear cytoplasmic pseudoinclusions, pale nuclei with powdery chromatin, and marginally placed micronucleoli. ${ }^{2,41}$ A recent study involving approximately 500 cases showed that the presence of 3 or more intranuclear pseudoinclusions is one of the best criteria to discriminate PTC from lesions classified as suspicious at cytology. ${ }^{41}$ Samples from FVPTC's demonstrate a microfollicular 
growth pattern with varying degrees of nuclear alterations (nuclear enlargement, membrane irregularities, and chromatin clearing). If the latter nuclear alterations associated with FVPTC are abundantly present in cytology samples, an accurate presurgical diagnosis of PTC may be rendered. Conversely, when these nuclear alterations are found in few cells, the specificity for malignancy also decreases because these alterations are observed in benign reactive conditions. In the explanatory notes on FVPTC, the TBSRTC states "current practice suggests that only the cases with definitive nuclear features of PTC should be diagnosed unequivocally on FNA". ${ }^{2}$ Because the threshold for making a cytopathological diagnosis of malignancy lies in the balance between the sensitivity to diagnose low-grade PTCs such as FVPTC and the specificity to minimize the risk of a false-positive diagnosis, the focus has shifted toward the interpretation of "definitive nuclear features". ${ }^{41,47}$

Molecular correlation is also indicated since in many studies the NIFTP group of lesions show "RAS-like" mutations and BRAF V600E mutations are almost non existent when the criteria for NIFTP are strictly applied ${ }^{41}$. (See further discussion in the molecular section).

\section{$\underline{\text { Papillary thyroid carcinoma }}$}

Papillary thyroid carcinoma (PTC) is the most common thyroid malignancy. The pathological diagnosis and classification of PTC is usually based on the microscopic appearance of biopsy specimens. Misdiagnosis of PTC by cytopathological methods has remained one of the most problematic issues in pathology. ${ }^{3}$ The pitfalls related to the cytological diagnosis of PTC should be analyzed in two parts concerning pattern and nuclear features. It is well known that nuclear details are the core of PTC diagnosis: "enlargement, oval shape, membrane irregularity, clearing, longitudinal grooves, intranuclear pseudoinclusions. Among the nuclear features of PTC, the most specific is intranuclear pseudoinclusions. ${ }^{41}$ One should be strict to define intranuclear pseudoinclusions, since their 
presence makes the aspirate at least suspicious for PTC. Intranuclear pseudoinclusions should be large enough and well-delineated with darker outlines to be discriminated from superimposed erythrocytes and artefacts. Nuclear grooves are usually present in PTCs, but to be reliable enough they should be widespread and clear rather than being a focal change or an artificial appearance caused by overlapping cells. Hypochromasia/nuclear clearing is an alerting finding, however depending on technical issues it might be misleading on its own. Uniformly enlarged oval shaped nuclei are also important in the diagnosis. ${ }^{1,3,4}$ The patterns of cytologic atypia mimicking PTC, formation of papillae or caps, are described in the literature. ${ }^{1,4}$ Pseudopapillary formation as an architectural pitfall is well document by Majahan et al. ${ }^{48}$ This study also stated that the presence of intranuclear cytoplasmic pseudoinclusions in parathyroid adenomas, not only overlaps with FN, but can also mimic PTC on aspirates. Psammoma bodies are quite specific for PTC; however dystrophic calcifications and dense colloid bodies should not be interpreted as psammoma bodies as these are almost never laminated in appearance in contrast to psammoma bodies. Another point is the amount and type of colloid that is usually scanty and of bubble-gum type in PTC. However, stromal degenerative fragments can mimic bubble-gum type colloid and large amounts of loose colloid may also be seen in PTC. Keeping in mind that all the cytological features cannot be present in each case one should be careful not to miss PTC; on the other hand, care is also needed not to overdiagnose nuclear and/or pattern atypia in benign entities as PTC. ${ }^{4}$ The two categories of the Bethesda System, "AUS/FLUS" and "suspicious for malignancy", are present for these equivocal findings and the degree of atypia determines the appropriate choice in reporting. ${ }^{2}$

Another primary thyroid tumour, which is frequently misdiagnosed as PTC or suspicious for PTC is hyalinizing trabecular tumour (HTT). It may also mimic MTC. Aspirates show oval to spindle-shaped cells with multiple intranuclear pseudoinclusions, in 
loose groups. Nuclei are uniform with smooth contours, grooves are uncommon. Presence of a metachromatic acellular material surrounded by tumour cells in a radiating fashion is one of the characteristics of this lesion. However, unfortunately this material may be interpreted as amyloid or colloid. Papillary or sheet-like fragments are generally absent and if immunocytochemistry is available the membrane staining (instead of the normal nuclei staining) for MIB-1 can be a helpful hint for HTT.4,49 The difficulty of a cytological diagnosis of HTT is underlined by the fact that according to a recent review of the literature, $60 \%$ of published cases of HTT in which a cytological diagnosis was included were misdiagnosed as suggestive, suspicious or positive for PTC. Of the remaining $40 \%$ of cases, $6 \%$ were reported as PTC versus HTT; $10 \%$ as follicular neoplasms (FNs); $1 \%$ as MTC; $10 \%$ as AUS; and, finally, only $8 \%$ of cases were correctly identified as HTT by FNA. Although architectural features are diminished in cytological samples, they are not absent, and must be taken into account and correctly interpreted when making a diagnosis. ${ }^{48}$ The absence of papillary structures and denuded fibrovascular stalks and the presence of elongated epithelial cells associated with acellular hyaline stroma are significant clues for HTT diagnosis. ${ }^{49}$

\section{$\underline{\text { Medullary thyroid carcinoma }}$}

Medullary thyroid carcinoma (MTC) is more aggressive than follicular derived neoplasms of the thyroid and is more frequently underdiagnosed than overdiagnosed. It is characterized by many single cells and loose tissue fragments; typically, follicular formation is not seen, and papillary structures are very rare. However, it may display nested/ trabecular/ loose microfollicular patterns, which could be misleading as follicular neoplasm, and may show oncocytic cytoplasm mimicking oncocytic follicular neoplasm. True oncocytes display centrally placed nuclei with macronucleoli which are unusual for MTC. ${ }^{1,4}$ MTC is one of the great mimickers of PTC; nuclear elongation, intranuclear pseudoinclusions, groove-like 
formations, and nuclear membrane irregularity are some of the cytomorphologic features that can be seen in both malignancies. However, a predominantly dispersed cell population, neuroendocrine type chromatin, some binucleation, eccentric nuclei, and presence of both plasmacytoid and spindle cells helps to identify MTC. Immunohistochemical studies and/or serum calcitonin analysis can give the final diagnosis. Rarely, some situations such as regenerative/degenerative processes with abundant spindle cells can mimic MTC. The nature of the chromatin and the clinical history and other laboratory examination results can help in the differential diagnosis. Paragangliomas are also a mimicker of MTC but to distinguish these entities by cytomorphology alone is nearly impossible, clinical findings and immunohistochemistry are needed. ${ }^{4}$ When amyloid is detected in thyroid FNA biopsies, MTC should be carefully searched for and excluded both by morphology and immunocytochemistry. ${ }^{4,50}$ The presence of amyloid can be a sign of primary or secondary amyloidosis (amyloid goitre) or can also accompany plasmacytomas and MALT lymphomas ${ }^{4,51}$. Due to its resemblance to dense colloid, recognizing amyloid on cytology slides may be difficult. Both amyloid and colloid are seen as metachromatic in Diff-Quick and blue-greenpink-orange in PAP stains; amyloid looks more solid and hyaline-like than colloid. ${ }^{4}$ The presence of amyloid may cause secondary inflammation, reactive changes and a giant cell reaction leading to an atypical/ suspicious diagnosis. ${ }^{4,51}$

\section{$\underline{\text { Metastatic tumours }}$}

Discussing the pitfalls in thyroid cytology, metastatic malignancies always should be kept in mind. It is always a possibility in the presence of a thyroid nodule, especially in patients with known malignancy in other organs. Any cytomorphology picture, which is not typical for common primary thyroid tumours should alert us to this possibility ${ }^{4}$. Metastasis from adjacent anatomical regions to the thyroid (pharynx, larynx, oesophagus, mediastinum, 
and regional lymph nodes) and distant ones sites are the most common.Lung, breast, skin (especially melanoma), colon, and kidney should be included in the differential diagnosis, particularly for some PTC and MTC variants and rarer tumours of thyroid ${ }^{2,4}$. However, distinguishing these entities by cytomorphology alone is nearly impossible as clinical findings and immunohistochemistry are needed. Immunocytochemistry can be applied in two steps. First, TTF-1, TTF-2, PAX8 or TG should be performed to show a primary thyroid origin of the tumour. After this step, if required further organ-specific markers can be performed in the light of the clinical information to detect the primary origin of the tumour ${ }^{2,4}$.

\section{Molecular tests and usefulness in false positive causes of thyroid FNA}

Besides, cytomorphological, radiological, and clinical findings, molecular tests have a place in patient management to overcome the inherent limitations of thyroid cytology. Molecular studies using cytological materials have gained increasing importance in recent years to decrease false positive rates and prevent unnecessary surgery ${ }^{52}$. According to the National Comprehensive Cancer Network guidelines, to gain a place in patient management for a molecular test the negative predictive value (NPV) should be $>95 \%$ as a rule out test, whereas the positive predictive value (PPV) should be $95 \%$ as a rule in test to enable operations such as total thyroidectomy ${ }^{53}$. Currently some rule in and rule out molecular tests are already in routine use especially in the USA in patient management ${ }^{53,54}$. A wide range of cytological material such as cell collection at R.O.S.E, cells obtained from slides in archive materials (air-dried slides and cell blocks) are suitable for use with the current molecular tests on the market ${ }^{53}$. For Category I/ND and Category II /Benign TBSRTC cases molecular analyses are not recommended in many guidelines, however, with the remarkable success of advances in molecular testing assays for point mutation analysis as well as chromosome rearrangements are already in use to tailor the management of indeterminate thyroid cytology lesions, namely AUS/FLUS, SFN/FN and SFM categories ${ }^{55}$. 
Molecular tests can be categorised as gene expression classifiers (GEC), gene mutational panels, targeted next-generation sequencing (tNGS) and combinations of mutation detection and miRNA expression These tests are used as both rule in and rule out tests ${ }^{56}$. Briefly, the main tests are:

AFIRMA: The microarray technology-based test Afirma GEC from Veracyte analyzes 167 different mRNA profiles composed of two sets of genes (25 genes for less common and 142 genes for the most common entities see in thyroid cancer). The test is validated as "rule out", i.e. "to say negative for samples cytomorphologically interpreted as negative" with the high negative predictive value (NPV) (95\% and 94\%, respectively) in AUS/FLUS and SFN/FN cases. On the other hand, NPV ratios are as low as $85 \%$ in the SFM category ${ }^{52,56}$.

ThyGenX/ ThyraMIR: ThyGenX (Asuragen) is a genomic mutation-based test using the advances in multiplexing analysis in cytology materials. This test, formerly known as the "seven gene test", was modified by Interpace Diagnostics as a thyroid oncogene panel. More than 100 genetic alterations of 8 genes associated with thyroid cancer (including PTC, FTC, MTC and ATC) can be detected. The test was designed as a "rule-in" test to detect malignancy in indeterminate lesions and a validation study to screen $B R A F, R A S, H R A S$, NRAS, PIK3CA point mutations, RET/PTC1, RET/PTC3, PAX8/PPAR $\gamma$ reported with $88 \%$, $87 \%$ and $95 \%$ PPV ratios for AUS/FLUS, SFN/FN and SFM diagnostic categories, respectively ${ }^{52,56}$

ThyraMIR, was developed by Interpace Diagnostics to search for 10 different miRNAs. NPV and PPV for malignancy were reported as $94 \%$ and $74 \%$. Although, these two tests can be used as separately, Interpace Diagnostics recommends ThyraMIR as a reflex test in the nodules that are shown to be negative by ThyGenX ${ }^{52,54}$. 
When both tests are negative, residual ROM can reduce up to 6\%. These tests have almost the same NPV ratios as AFIRMA, besides the high PPV ratios, and therefore are acceptable as "rule in" and "rule out" tests ${ }^{52}$.

ThyroSeq TEST: A NGS-based gene mutation and fusion panel that has been designed to detect 12 cancer genes and 284 mutational hot spots. In 2014, an enhanced version was introduced to the market with DNA (14 genes and >1000 mutations) and RNA (42 fusions, 16 genes) alterations. The study of Nikiforov et al. showed that ThyroSeq is a good "rule-out" test with the 98\% NPV ratios, whereas PPV ratios were found between 40\%-69\% (a high rate of false positives possibly being due to the expanded NGS-based mutational profile) which is not a promising as a "rule-in" test. Recently, same group released a validation study of a ThyroSeq v3 genomic classifier that analyzes 112 genes for various genetic alterations (point mutations, insertions/deletions, gene fusions, copy number alterations, and abnormal gene expressions). ThyroSeq v3 showed high accuracy for all common thyroid cancer types as well as parathyroid lesions. By adding copy number analyses, ThyroSeq v3 claimed improved accuracy for Hurthle cell tumours $52,54,56,57$.

BRAF v $600 E$ mutation: $B R A F$ V600E mutation is known to be $>99 \%$ specific for diagnosis of thyroid cancer (particularly for PTC), therefore mutational positivity of BRAF V600E is meaningful when cytomorphological findings indicate an indeterminate cytology result. BRAF V600E testing is also useful in NIFTP diagnosis, since these tumours are follicular derived $R A S$-driven thyroid lesions and almost never show BRAF V600E mutations if strict morphological criteria for NIFTP are applied. When the strict diagnostic criteria of NIFTP are applied, the presence of a BRAF V600E mutation should therefore exclude a NIFTP tumour in FNA aspirates associated with the subtle nuclear changes of PTC with a very high degree of certainty $^{58}$. 


\section{Medicolegal Implications}

The expectation of patients and clinicians is that thyroid FNA will work as an effective diagnostic test, but given its limitations, it is inevitable that false positive cytological diagnoses of malignancy will occur in thyroid FNA cytology. A recent survey of medicolegal claims against the NHS Litigation Authority for thyroid surgery in England in the period 1995-2012 showed 223 claims related to thyroid surgery of which 22 cases related to diagnostic delay or incorrect diagnosis, many of which inevitably involve pathology or cytology ${ }^{59}$.In thyroid FNA cytology the interobserver and also intraobserver differences in diagnosis that are seen in routine clinical practice between a TBSRTC category VI/Thy 5 diagnosis and TBSRTC category V/Thy 4 are frequently quite subjective and are often a matter of professional opinion among trained and experienced cytopathologists rather than a matter of fact. These differences can be readily explained by the known interobserver variation in the reporting of thyroid FNAC. For example in an interobserver study among 6 experienced cytopathologists of 200 randomly selected thyroid FNA specimens using the UK Royal College of Pathologists Thyroid FNA Classification the number of cases diagnosed as Thy 5 was mean 18.6 (range 8-23) and Thy 416.6 (range 8-26) ${ }^{60}$. Cibas and colleagues later showed using the 6 category Bethesda system that only $64.0 \%$ of diagnoses made by local cytopathologists and a central review panel of cytopathologists were concordant. In this study only $74.7 \%$ of intraobserver diagnoses were concordant when the same slides were reviewed blindly on a different occasion by the same cytopathologist. Concordance of central review panel of cytopathologists with local cytopathologists for the malignant TBSRTC category VI was $94 \%(n=51)$ and for suspicious for malignancy TBSRTC category V, 37\% $(n=38)^{61}$. Hence in this major study $6 \%$ of cases that were diagnosed as TBSRTC category VI (equivalent to Thy 5 in the UK) by local cytopathologists were downgraded on central cytopathology review. Since the risk of malignancy for TBSRTC category VI is less than 
$100 \%$, patients need to be aware of the risk of malignancy and also the false negative risk in this category and a disclaimer about the risk of malignancy of each category can be presented in the cytology reports to inform both patients and their surgeons.

\section{$\underline{\text { Conclusions }}$}

The main role of thyroid FNAC is the triage of patients who actually require surgery, thus reducing the number of unnecessary thyroidectomies and possible complications. ${ }^{62}$ Ultrasound image features must be taken into account to plan appropriate patient management in a multidisciplinary approach to patient management. A cytology result with suspicious or malignant findings in the presence of a nodule with benign ultrasound features should be carefully studied to avoid a false positive diagnosis. As a cytological diagnosis of malignancy may require aggressive surgical treatment, including total thyroidectomy with possible central neck dissection, the correct FNA diagnosis is important to guide proper management of patients and to avoid overtreatment. In some cases if there is still uncertainty cell block immunohistochemistry of the current specimen or of a repeat thyroid FNAC and/or core biopsy will allow confirmation of the presence of malignancy and also the exact nature of the malignancy (e.g. medullary carcinoma or poorly differentiated carcinoma) . Molecular testing including for BRAF V600E mutation may also be helpful. All centres should audit their own practice of both thyroid cytology and histology to identify the risk of malignancy for all subcategories of thyroid FNAC, whether using TBSRTC, UK RCPath Thy or another internationally recongised terminology system bearing also in mind that the histopathological diagnosis may also be subject to significant interobserver variation ${ }^{62 .}$ It is important to note that an accurate diagnosis by FNA crucially depends on the sample being representative and adequate in cellularity, appropriately processed, and stained, in order to provide high-quality 
preparations for assessment. When these conditions are not met, repeat sampling with or without on-site assessment should be considered prior to surgical management.

\section{$\underline{\text { References }}$}

1. Schmitt F. Cytopathology. In Encyclopedia of Pathology. Cham, Springer, 2017.

2. Ali SZ, Cibas ES. The Bethesda System for Reporting Thyroid Cytopathology: Definitions, Criteria and Explanatory Notes. $2^{\text {nd }}$ Ed. Cham, Springer, 2018.

3. Yi K, Ahn S, Park DY et al. False-positive cytopathology results for papillary thyroid carcinoma: a trap for thyroid surgeons. Clinical Otolaryngology 2017; 42: 1153-1160.

4. Canberk S, Firat P, Schmitt F. Pitfalls in the cytological assessment of thyroid nodules. Turkish J Pathol 2015; 31:18-33.

5. Cross P, Chandra A, Giles T, Johnson S, Kocjan G, Poller D, Stephenson T. Guidance on the reporting of thyroid cytology specimens. The Royal College of Pathologists. January 2016 6. Chadwick D, Kinsman R, Walton P. Fifth National Audit Report. The British Association of Endocrine \& Thyroid Surgeons (accessed 11.4.18)

7. Durante C, Grani G, Lamartina L, Filetti S, Mandel SJ, Cooper DS. The diagnosis and management of thyroid nodules: a review. JAMA 319;:914-24.

8. Ashcraft MW, Van Herle AJ. Management of thyroid nodules. II: scanning techniques, thyroid suppressive therapy, and fine needle aspiration. Head Neck Surg 1981; 3: 297-322.

9. Gharib H, Goellner JR. Fine-needle aspiration biopsy of the thyroid: an appraisal. Ann Intern Med 1993; 118:282-9.

10. Corrias A, Einaudi S, Chiorboli E, Weber G, Crino A, Andreo M, et al. Accuracy of fine needle aspiration biopsy of thyroid nodules in detecting malignancy in childhood: comparison with conventional clinical, laboratory, and imaging approaches. J Clin Endocrinol Metab 2001; 86:4644-8. 
11. Arda IS, Yildirim S, Demirhan B, et al. Fine needle aspiration biopsy of thyroid nodules. Arch Dis Child 2001; 85:313-7.

12. Amrikachi M, Ponder TB, Wheeler TM, et al. Thyroid fine-needle aspiration biopsy in children and adolescents: experience with 218 aspirates. Diagn Cytopathol 2005; 32:189-92.

13. Hosler GA, Clark I, Zakowski MF, et al. Cytopathologic analysis of thyroid lesions in the pediatric population. Diagn Cytopathol 2006; 34:101-5.

14. Stevens C, Lee JK, Sadatsafavi M, Blair GK. Pediatric thyroid fine-needle aspiration cytology: a meta-analysis. J Pediatr Surg. 2009;44:2184-91.

15. Lew JI, Snyder RA, Sanchez YM, Solorzano CC. Fine needle aspiration of the thyroid: correlation with final histopathology in a surgical series of 797 patients. J Am Coll Surg 2011; 213:188-194.

16. Bongiovanni M, Spitale A, Faquin WC et al.The Bethesda System for Reporting Thyroid Cytopathology: a meta-analysis. Acta Cytol. 2012; 56:333-339.

17. Richmond BK, Judhan R, Chong B, et al. False-negative results with the Bethesda System of reporting thyroid cytopathology: predictors of malignancy in thyroid nodules classified as benign by cytopathologic evaluation. Am Surg. 2014; 80:811-816.

18. McHenry CR, Slusarczyk SJ, Khiyami A. Recommendations for management of cystic thyroid disease. Surgery 1999; 126:1167-1172.

19. Faquin WC, Cibas eS, Renshaw aa. "atypical" cells in ne- needle aspiration biopsy specimens of benign thyroid cysts. Cancer. 2005; 105:71-9.

20. Jaragh M, Carydis Vb, MacMillan C, Freeman J, Colgan TJ. Predictors of malignancy in thyroid ne-needle aspirates "cyst fluid only" cases: Can potential clues of malignancy be identified? Cancer. 2009; 117:305-10. 
21. Jaffar R, Mohanty SK, Khan A, Fischer AH. Hemosiderin laden macrophages and hemosiderin within follicular cells distinguish benign follicular lesions from follicular neoplasms. Cytojournal. 2009; 6:3.

22.Faquin WC, Cibas ES, Renshaw AA. “Atypical” cells in fine needle aspiration biopsy specimens of benign thyroid cysts. Cancer. 2005; 105:71-9.

23. Sharma C, Krishnanand G. Histologic analysis and comparison of techniques in fine needle aspiration-induced alterations in thyroid. Acta Cytol 2008; 52: 56-64.

24.Gage H, Hubbard E, Nodit L. Multiple squamous cells in thyroid fine needle aspiration: friends or foes? Diagn Cytopathol 2016; 44: 676-681.

25. Anderson SR, Mandel S, Livolsi VA, Gupta PK, Baloch ZW. Can cytomorphology differentiate between benign nodules and tumors arising in Graves' disease? Diagn Cytopathol. 2004; 31:64-7.

26. Solano JG, et al. Fine-needle aspiration of subacute granulomatous thyroiditis (De Quervain's thyroiditis): a clinico-cytologic review of 36 cases. Diagn Cytopathol. 1998; 16:214-220.

27. Singer PA. Thyroiditis. Acute, subacute and chronic. Med Clin North Am. 1991; 75:6177.

28. Oláh R, Hajós P, Soós Z, Winkler G. De Quervain thyroiditis. Corner points of the diagnosis. Orv Hetil. 2014; 155:676-80.

29. Lamichaney R, Sherpa M, Das D, Bhutia CT, Laishram S. Fine-Needle Aspiration of De Quervain's Thyroiditis (Subacute Granulomatous Thyroiditis): A Cytological Review of 20 Cases. J Clin Diagn Res. 2017; 8: EC09-EC11. 
30. Anila KR, Nayak N, Jayasree K. Cytomorphologic spectrum of lymphocytic thyroiditis and correlation between cytological grading and biochemical parameters. Journal of Cytology. 2016; 33:145-149.

31. Harvey AM, Truong LD, Mody DR. Diagnostic pitfalls of Hashimoto's/lymphocytic thyroiditis on fine-needle aspirations and strategies to avoid overdiagnosis. Acta Cytol. 2012; 56:352-60.

32. Stacchini A, Demurtas A, Aliberti S. Extranodal lymphoproliferative processes and flow cytometry. Acta Cytol 2016; 60: 315-325.

33.Anderson SR, Mandel S, LiVolsi VA et al. Can cytomorphology differentiate between benign nodules and tumors arising in Graves’ disease? Diagn Cytopathol. 2004;31:64-67.

34.Mazeto G, Brandão J, Pimenta W et al. Revisão de 2564 punções aspirativas de tireóide: ênfase em doenças não neoplásicas. J Bras Patol 1998; 34:39-47.

35. Yeung MJ, Serpell JW. Management of the solitary thyroid nodule. Oncologist 2008; 13:105-12.

36. Baloch ZW, LiVolsi VA. Follicular-patterned afflictions of the thyroid gland: Reappraisal of the most discussed entity in endocrine pathology. Endocr Pathol. 2014; 25:12-20.

37. Renshaw AA, Wang E, Wilbur D, Hughes JH, Haja J, Henry MR. Interobserver agreement on microfollicles in thyroid fine needle aspirates. Arch Pathol Lab Med. 2006; 130: $148-52$.

38. Nikiforov YE, Seethala RR, Tallini G, et al. Nomenclature revision for encapsulated follicular variant of papillary thyroid carcinoma: a paradigm shift to reduce overtreatment of indolent tumors. JAMA Oncol. 2016; 2: 1023-1029.

39. Strickland KC, Vivero M, Jo VY, et al. Preoperative cytologic diagnosisof noninvasive follicular thyroid neoplasm with papillary-like nuclear features: a prospective analysis. Thyroid. 2016; 26: 1466-1471. 
40. Canberk S, Baloch Z, Ince U, Schmitt F. Diagnosis of Non-invasive Follicular Tumor with Papillary-like Nuclear Features (NIFTP): A Practice Changer for Thyroid Fine-needle Aspiration Interpretation. Journal of Basic and Clinical Medicine 2017; 6: 38-43.

41. Ohori NP, Wolfe J, Carty S et al. The influence of the Noninvasive Follicular Thyroid Neoplasm with Papillary-like Nuclear Features (NIFTP) resection diagnosis on the falsepositive thyroid cytology rate relates to quality assurance thresholds and the application of NIFTP criteria. Cancer Cytopathol 2017; 125: 692-700.

42. Agarwal AM, Bentz JS, Hungerford R, Abraham D Parathyroid fine-needle aspiration cytology in the evaluation of parathyroid adenoma: Cytologic findings from 53 patients. Diagn Cytopathol. 2009; 37:407-10.

43. Plamper M, Schreiner F, Gohlke B et al. Thyroid disease in children and adolescents with PTEN hamartoma tumor syndrome (PHTS). Eur J Pediatr 2018; 177: 429-435.

44. Maximo V, Rios E, Sobrinho-Simoes M. Oncocytic lesions of the thyroid, kidney, salivary glands, adrenal cortex and parathyroid glands. Int J Surg Pathol. 2014; 22:33-36 45. Montone KT, Baloch ZW, LiVolsi VA. The thyroid Hürthle (oncocytic) cell and its associated pathologic conditions: A surgical pathology and cytopathology review. Arch Pathol Lab Med. 2008; 132:1241-50.

46. Kasper KA, Stewart J, Das K. Fine-Needle Aspiration Cytology of Thyroid Nodules with Hürthle Cells: Cytomorphologic Predictors for Neoplasms, Improving Diagnostic Accuracy and Overcoming Pitfalls. Acta Cytol 2014; 58:145-152.

47. Mito JK, Alexander EK, Angell TE et al. A modified reporting approach for thyroid FNA in the NIFTP era: A 1-year institutional experience. Cancer Cytopathol 2017; 125: 854-64.

48. Mahajan A, Lin X, Nayar R. Thyroid Bethesda reporting category, 'suspicious for papillary thyroid carcinoma', pitfalls and clues to optimize the use of this category. Cytopathology 2013; 24:85-91. 
49. Saglietti C, Piana S, La Rosa S, Bongiovanni M. Hyalinizing trabecular tumour of the thyroid: fine-needle aspiration cytological diagnosis and correlation with histology. J Clin Pathol 2017; 70: 641-647.

50. Trimboli P, Treglia G, Guidobaldi L et al. Detection rate of FNA cytology in medullary thyroid carcinoma: a meta-analysis. Clin Endocrinol 2015; 82: 280-285.

51. Ozdemir BH, Uyar P, Ozdemir FN Diagnosing amyloid goitre with thyroid aspiration biopsy. Cytopathology. 2006; 17: 262-6.

52. Zhang M, Lin O. Molecular Testing of Thyroid Nodules: A Review of Current Available Tests for Fine-Needle Aspiration Specimens. Arch Pathol Lab Med. 2016 140:1338-1344.

53. Tuttle RM, Haddad RI, Ball DW, et al: Thyroid carcinoma, version 2.2014. J Natl Compr Canc Netw 2014;12:1671-1680.

54. Paschke R, Cantara S, Crescenzi A, Jarzab B, Musholt TJ, Sobrinho Simoes M.European Thyroid Association Guidelines regarding Thyroid Nodule Molecular Fine-Needle Aspiration Cytology Diagnostics. Eur Thyroid J. 2017; 6:115-129.

55. Ferris RL, Baloch Z, Bernet V, et al. American thyroid association statement on surgical application of molecular profiling for thyroid nodules: current impact on perioperative decision making. Thyroid. 2015; 25:760-768.

56. Poller DN, Glaysher S. Molecular pathology and thyroid FNA. Cytopathology. 2017 28:475-481

57. Nikiforova MN, Mercurio S, Wald AI et al .Analytical performance of the ThyroSeq v3 genomic classifier for cancer diagnosis in thyroid nodules. Cancer. 2018; doi: 10.1002/cncr.31245. [Epub ahead of print] 
58. Basolo F, Macerola E, Ugolini C, Poller DN, Baloch Z. The Molecular Landscape of Noninvasive Follicular Thyroid Neoplasm With Papillary-like Nuclear Features (NIFTP): A Literature Review. Adv Anat Pathol. 2017; 24:252-258.

59. Dent PC, Bagnall NM. Litigation in thyroid surgery in England. British Journal of Hospital Medicine 2017;78: 213-218

60. Kocjan G, Chandra A, Cross PA, et al The interobserver reproducibility of thyroid fine needle aspiration using the UK Royal College of Pathologists classification system. Am J Clin Pathol 2011;135:852-859

61. Cibas ES, Baloch ZW, Fellegara G et al. A prospective assessment defining the limitations of thyroid nodule pathologic evaluation. Ann Intern Med 2013;159:325-332

62. Tallini G, Gallo C. Fine-needle aspirations and intraoperative consultation in thyroid pathology: when and how? Int J Surg Pathol 2011; 19: 141-144. 


\section{Legend for figures}

Figure 1. Reactive cyst lining cells with elongated cytoplasm showing nuclei with some irregular contours (MGG, 40X).

Figure 2. Reactive epithelial follicular and histiocytic cells with overlapping due smear artefact in case of granulomatous thyroiditis (MGG, 20X).

Figure 3. Cell debris in background of smear of thyroiditis simulating necrosis (MGG, 10X).

Figure 4. Atypical oncocytes in Hashimoto thyroiditis. Note presence of lymphocytes. (MGG, 40X).

Figure 5. Florid lymphoid phase of Hashimoto thyroiditis simulating a low-grade lymphoma. Most of these cases need flow cytometry for the definitive diagnosis. (MGG, 40X)

Figure 6. Pseudo microfollicular appearance create by the presence of clots (PAP, 20X). 